\title{
Density functional approach to study structural properties and Electric Field Gradients in rare earth materials
}

\author{
S. Jalali Asadabadi* \\ Department of Physics, Faculty of Science, University of Isfahan (UI) \\ Hezar Gerib Avenue, Isfahan 81744, Iran and \\ Research Center for Nano Sciences and Nano Technology \\ University of Isfahan (UI), Isfahan 81744, Iran \\ H. Akbarzadeh $\dagger$ \\ Department of Physics \\ Isfahan University of Technology \\ Isfahan, Iran
}

(Dated: October 29, 2018)

\begin{abstract}
We investigated the effect of spin polarization on the structural properties and gradient of electric field (EFG) on $\mathrm{Sn}$, In, and $\mathrm{Cd}$ impurity in $\mathrm{RSn}_{3}$ ( $\mathrm{R}=\mathrm{Sm}, \mathrm{Eu}$, Gd) and $\mathrm{RIn}_{3}(\mathrm{R}=\mathrm{Tm}, \mathrm{Yb}, \mathrm{Lu})$ compounds. The calculations were performed selfconsistently using the scalar-relativistic full potential linearized augmented plane wave method. The local density approximations (LDA) and generalized gradient approximation without spin polarization (GGA) and with spin polarization (GGA+SP) to density functional theory were applied. In addition to that we performed some calculations within open core treatment ( GGA+open core). It is clearly seen that $\mathrm{GGA}+\mathrm{SP}$ is successful in predicting the larger lattice parameter and the dramatic drop of $\mathrm{EFG}$ for $\mathrm{R}=(\mathrm{Eu}, \mathrm{Yb})$ relative to other rare earth compounds. This is an indication that spin splitting generated by spin polarization without any modification, is capable of treating properly the highly correlated f electrons in these systems.
\end{abstract}

\section{INTRODUCTION}

The highly correlated f electrons in rare earth materials have challenged the reliability of the results obtained by conventional density functional theory (DFT). It is generally believed that regular DFT in its LDA/GGA formulation, without any modification, is in-

${ }^{*}$ Electronic address: sjalali@phys.ui.ac.ir

${ }^{\dagger}$ Electronic address: akbarzad@cc.iut.ac.ir 
sufficient to correctly deal with the f electrons; hence our understanding of the physical properties of these systems are often based on a many body model Hamiltonian or modified LDA such as LDA+U [1, 2, 3], LDA+SIC 14, 5], and LDA+(open core) [6] approaches.

Strange et.al [5], by removing the spurious self-interaction of $\mathrm{f}$ electrons reported a systematic investigation of the rare earth elements and their sulfides. They confirmed the pre-claimed idea that there are two types of $\mathrm{f}$ electrons in these materials: Localized core-like $f$ electrons that determine the valency, and delocalized band-like $f$ electrons that are formed through hybridization with the s-d bands and which participate in bonding. The main feature of their calculation was based on an $\mathrm{f}$ band splitting into two sub-bands one occupied, and the other unoccupied.

In a recent publication we investigated the valency of rare earths in $\mathrm{RIn}_{3}$; $(\mathrm{R}=\mathrm{Sm}$, $\mathrm{Eu}, \mathrm{Gd})$ and $\mathrm{RSn}_{3}$; (R=Tm, $\left.\mathrm{Yb}, \mathrm{Lu}\right)$ and calculated the electric field gradient using DFT without spin polarization [7]. We found out that in regular DFT as all $\mathrm{f}$ electrons are treated as band electrons, consequently their density of states are all located in a single peak at the fermi level which is not consistent with reality. We finally overcame this drawback by applying open core approach.

The aim of the present work is to investigate the effect of spin polarization on structural properties and the gradient of electric field of the same samples. Our objective is to investigate the borderline of LDA ability, that without any modification further than spin polarization can obtain reliable results for some compounds with highly correlated f electrons. Our calculations confirm the fact that in most cases spin polarization, without using LDA+U, LDA+SIC, or even open core modification can treat the highly correlated $\mathrm{f}$ electrons properly. It means that at low temperature, where the system is magnetically ordered, spin polarization, by providing more flexibility among f electrons and opening degrees of freedom for spin-splitting, partly compensate the necessary coulomb correlation.

The organization of this paper is as follow. In Section II we present the calculational details. Section III deals with the results and discussions. Finally, we conclude this work with a summary in Section IV. 


\section{CALCUlational Details}

We utilize the full potential linearized augmented plane wave (FP-LAPW) method within density functional theory. In this method wavefunctions, charge density, and potential are expanded in spherical harmonics within nonoverlapping muffin-tin spheres and in plane waves in the remaining interstitial region of the unit cell . As in FP-LAPW method no shape approximation is introduced for potential and charge density, then it is an appropriate procedure to obtain reliable results for the electric field gradient (EFG) with high sensitivity to unisotropic charge density distribution around the nucleus.

The EFG tensor is defined as the second derivative of the coulomb potential with respect to the Cartesian coordinate at the nucleus, which is written as a traceless tensor. The coulomb potential which is one of the crucial quantities of an accurate (fullpotential) band structure calculation is given in LAPW as lattice harmonics expansion inside the atomic spheres. In this representation, the required derivatives can be determined straightforwardly, and the largest component of EFG tensor can be obtained directly from the $\mathrm{L}=2, \mathrm{M}=0$ component of the potential expansion inside the muffin tin spheres|8]:

$$
V_{z z}=\sqrt{\frac{5}{4 \pi}} \lim _{r \rightarrow 0} \frac{V_{20}}{r^{2}}
$$

It is assumed that the main axis of the EFG tensor points towards $z$ direction. The radial potential coefficient $V_{20}(r)$ near the nucleus $(r \rightarrow 0)$ for a given charge density is obtained numerically by solving Poisson's equation using a method proposed by Weinert [9].

The calculations were performed using the WIEN97 code developed by Blaha and coworkers [10], which has been successfully applied to a wide range of systems. In this code core and valence states separated by their energy are treated differently. Core states are treated within multiconfiguration relativistic Dirac-Fock approach while valence states are only scalar relativistic with an exception of $E u S n_{3}$ for which the spinorbit coupling in a second order variational steps were included during self-consistent calculations of valence state. In the later case the number of symmetry was reduced from 48 to 16 by assuming $z$-direction as a preferentional direction of the coupled spin to the orbit. It was shown that by including the spin-orbit interaction the results does not change substantially, and as the incorporation of this coupling is very time consum- 
ing then it was neglected throughout the calculation. For better treatment of the core electrons we decomposed them into high lying semicore and deeper true core states. As the electronic charge of semicore states are not completely confined inside the respective muffin-tin spheres, therefore we treated them as band states in the full (nonspherical) potential. For these states in addition to the usual LAPW basis we included local orbitals to increase the flexibility of the basis set [11], and being able to use a sufficiently fine $\mathrm{k}$ sampling. This procedure results the more accurate calculation of EFG, which is extremely sensitive to the basis set [12]. The true core states were treated atomic like in the self-consistent muffin-tin potential. In our calculations electronic states with an energy of at least 6 Ry below the Fermi energy were considered as true core states. For the muffin-tin radius of rare earth elements as well as $\mathrm{Sn}$ and $\mathrm{In}$ a value of 2.65 a.u. were chosen throughout while for the Cd impurity manipulated into the $\mathrm{RSn}_{3} / \mathrm{In}_{3}$ compounds a radius of $2.5 \mathrm{a} . \mathrm{u}$. was used. The maximum angular momentum quantum number 1 as a cutoff for expanding the Kohn-Sham wavefunctions in terms of lattice harmonics inside the muffin-tin spheres was confined to $l_{\max }=12$. The potential and charge density was expanded inside the atomic spheres in crystal harmonics up to $\mathrm{L}=6$. The wavefunctions in the interstitial region were expanded in plane waves with a cutoff of $K_{\max }=7 / R_{M T}$ where $R_{M T}$ is the smallest muffin-tin radius in the unit cell. The periodic charge density and potential were Fourier expanded up to $G_{\max }=16$. We adjusted the value of mixing parameter of charge density to 0.1 and used Broyden's scheme. We have taken a mesh of 165 special $\mathrm{k}$ points to implement the valence state integration in the irreducible wedge of the Brillioun zone (BZ) that correspond to the grids $18 \times 18 \times 18$ in the scheme of Monkhorst-Pack [13]. The mesh of k points, charge density and potential expansion cutoff and finally muffin-tin radii were allowed to vary for ensuring the convergence. The exchange-correlation energy was calculated within the local density approximation (LDA) based on the Perdew-Zunger parameterization [14], with and without including advanced generalized gradient approximation (GGA) using PerdewBurke-Ernzerhof parameterization [15], with and without considering spin polarization. For spin polarized calculations, a ferromagnetic moment was imposed on $\mathrm{R}$ at the start of the selfconsistency cycle. As for some compounds this is not the true magnetic order [16], such an assumtion should be considered as an extra possible source of error. At the end of selfconsistency cycle, $\mathrm{YbIn}_{3}$ and $\mathrm{LuIn}_{3}$ came out to be nonmagnet, all others remained ferromagnetic. In order to calculate the electric field gradient at $\mathrm{Cd}$ site as 
TABLE I: The comparison of the measured lattice parameters in $\AA$ at $\mathrm{T}=300 \mathrm{~K}$ with the theoretical values calculated at $\mathrm{T}=\mathrm{OK}$ within LDA, GGA without spin polarization (GGA), GGA with spin polarization (GGA+SP), and GGA+open core (valence 2, 2.5, 3).

\begin{tabular}{ccccccc}
\hline \hline & $\mathrm{SmSn}_{3}$ & $\mathrm{EuSn}_{3}$ & $\mathrm{GdSn}_{3}$ & $\mathrm{TmIn}_{3}$ & $\mathrm{YbIn}_{3}$ & $\operatorname{LuIn}_{3}$ \\
\hline LDA & 4.529 & 4.515 & 4.513 & 4.434 & 4.443 & 4.434 \\
GGA & 4.654 & 4.649 & 4.648 & 4.589 & 4.594 & 4.566 \\
GGA+SP & 4.708 & 4.715 & 4.683 & 4.593 & 4.594 & 4.566 \\
$G G A^{\text {divalent }}$ & 4.809 & 4.784 & 4.749 & 4.651 & 4.626 & 4.655 \\
$G G A^{\text {di \&halfvalent }}$ & - & 4.758 & 4.733 & 4.619 & 4.604 & - \\
$G G A^{\text {trivalent }}$ & 4.747 & 4.732 & 4.717 & 4.587 & 4.582 & 4.576 \\
a Expt. & 4.69 & 4.75 & 4.68 & 4.56 & 4.61 & 4.55 \\
\hline \hline
\end{tabular}

${ }^{a}$ Ref.[16, 20]

a representation of an impurity atom in each compound all sides of the unit cell were doubled to construct a supercell with 16 atoms [? ]. Further increase of the sides of supercell did not change the results significantly. To keep the same accuracy with the previous primitive unit cell the mesh of $\mathrm{k}$ points in supercell was reduced to 75 corresponding to the $9 \times 9 \times 9$ grids. The energy for each orbital in $E u S n_{3}$, and in $\operatorname{YbIn}_{3}$ is linearized by selecting the most probable energy calculated for each density of state over the valence and semicore energy windows. The linearizations obtained for $E u S n_{3}$ and $Y b{ }_{3} n_{3}$ then are used as an initial input for the $S m S n_{3}, G d S n_{3}$, and the $T m I n_{3}, \operatorname{LuIn}_{3}$, respectively and we allow the program to search automatically for the best-linearized energies of Cd orbitals and check if they are perfect.

\section{RESULTS AND DISUSION}

\section{A. Structural Properties}

By calculating the total energy of a primitive unit cell as a function of its volume and fitting the data with the Birch equation of state [17] we obtained the lattice parameters, bulk moduli, and the pressure derivative of the bulk moduli for $\mathrm{RSn}_{3}$ ( $\left.\mathrm{R}=\mathrm{Sm}, \mathrm{Eu}, \mathrm{Gd}\right)$ and $\mathrm{RIn}_{3}(\mathrm{R}=\mathrm{Tm}, \mathrm{Yb}, \mathrm{Lu})$ by LDA, GGA, GGA+SP, GGA +open core (valence 2, 2.5 and 3) approaches. These results along with the experimental data are listed in Tables Iand II.

The lattice parameters calculated by GGA for all compounds are in better agreement 
with the experiment than those obtained by LDA. The better prediction of experimental results by GGA has also been observed in most rare earth metals and is believed to be due to the fact that the nonlocality of exchange and correlation is better taken into account by GGA than LDA [18]. We expect the lattice constant for divalent $\mathrm{Eu}$ and $\mathrm{Yb}$ to be higher than the other rare earth compounds. The divalent character results to weaker interatomic bonds and manifests itself in a higher lattice parameter and also compressibility, which is the inverse of bulk modulus. It is clearly seen that GGA fails to predict such behavior for Eu compound, while GGA+SP improves the results in the right direction. Richter [6] by comparing the charge densities belonging to non-magnetic and polarized solutions of 4 f electrons of gadolinium atom has illustrated a clear reason why spin-independent DFT is not suited to magnetic systems. Our results confirm his conclusions. As expected, due to rather full $\mathrm{f}$ shell in $\mathrm{Lu}, \mathrm{Yb}$ and even $\mathrm{Tm}$ compounds, spin polarization does not practically affect their lattice parameter. For $S m S n_{3}$ and $G d S n_{3}$ the results obtained by GGA+SP are in nice agreement with experiment while for $\mathrm{Eu}$ and $\mathrm{Yb}$ compounds the results obtained by open core calculation with a mixed valence $(\sim 2.5)$ are in better agreement with experiment. A point worth to mention is that the deviation of lattice parameter calculated by GGA+SP and GGA+open core are more significant in $\mathrm{Sm}, \mathrm{Eu}$, and $\mathrm{Gd}$ compared to $\mathrm{Tm}, \mathrm{Yb}$, and $\mathrm{Lu}$ compounds. It is probably because of the fact that going from left to right in the list of lantanides the $f$ electrons are getting more localized. Hence, for the highly localized $\mathrm{f}$ electrons in Tm, $\mathrm{Yb}$, and Lu compounds it does not make any difference if we leave them alone or confine them inside the core by open core approach. Similar behavior has been predicted for $\mathrm{f}$ electrons in actinides [19]. Finally, it should be noticed that although the equilibrium lattice parameters can be accurately extracted by fitting the total energy-volume data with an appropriate equation of state, but the value obtained for the bulk modulus and its pressure derivative are sensitive to the range of fitting. Hence, for the results presented in Table $\amalg$ only the qualitative analysis are reliable and we can not fully trust the quantitative values. Furthermore as to our knowledge no experimental data for the bulk modulus and its derivative has been reported yet, hence our results serve only as a prediction for future studies. 
TABLE II: The calculated Bulk modulus in GPa and its pressure derivative within LDA, GGA without spin polarization (GGA), and GGA with spin polarization (GGA+SP).

\begin{tabular}{ccccccc}
\hline \hline & $\mathrm{SmSn}_{3}$ & $\mathrm{EuSn}_{3}$ & $\mathrm{GdSn}_{3}$ & $\mathrm{TmIn}_{3}$ & $\mathrm{YbIn}_{3}$ & $\mathrm{LuIn}_{3}$ \\
\hline$B_{0}(\mathrm{LDA})$ & 65.517 & 59.786 & 62.549 & 73.032 & 70.596 & 77.468 \\
$B_{0}^{\prime}(\mathrm{LDA})$ & 2.509 & 2.312 & 2.948 & 5.789 & 5.089 & 4.708 \\
$B_{0}$ (GGA) & 63.531 & 61.020 & 59.629 & 53.440 & 55.180 & 62.604 \\
$B_{0}^{\prime}(\mathrm{GGA})$ & 3.478 & 3.401 & 3.560 & 4.937 & 4.486 & 4.159 \\
$B_{0}(\mathrm{GGA}+\mathrm{SP})$ & 58.011 & 54.239 & 64.318 & 55.788 & 55.081 & 62.648 \\
$B_{0}^{\prime}(\mathrm{GGA}+\mathrm{SP})$ & 4.354 & 5.064 & 4.688 & 4.709 & 4.402 & 4.155 \\
\hline \hline
\end{tabular}

\section{B. Electric Field Gradient}

In the literature, different conversion relations analyze the experimental results of the nuclear quadrupole interaction. Then to compare our calculated EFG with experiment we first need to specify the conversion relation that has been used. The experimental data to calculate the EFG on $\mathrm{Sn}$ site of $\mathrm{RSn}_{3}$; $(\mathrm{R}=\mathrm{Sm}, \mathrm{Eu}, \mathrm{Gd})$ compounds are obtained from Mossbauer spectroscopy. Hence the value of $V_{z z}$ can be calculated from the measured Doppler velocity $\Delta_{\nu}$ via :

$$
V_{z z}\left[10^{21} V m^{-2}\right]=12.79 \Delta_{\nu}\left(m m s^{-1}\right)
$$

While the EFG on a Cd impurity substituted at Sn and In sites were obtained by Perturbed Angular Correlation measurements with the following conversion relation:

$$
V_{z z}\left[10^{21} \mathrm{Vm}^{-2}\right]=\frac{0.041355}{Q(b)} \omega(M H z)
$$

We used the recently proposed value of $\mathrm{Q}=0.83 \mathrm{~b}$ for nuclear quadrupole moment of $\mathrm{Cd}$.

The calculated EFG on $\mathrm{Sn}$ sites of $\mathrm{RSn}_{3}$; $(\mathrm{R}=\mathrm{Sm}, \mathrm{Eu}, \mathrm{Gd})$ are compared with the experimental data in Table III. For all compounds, the less agreement with experiment is due to LDA calculations. GGA + SP correctly predict the lower value of EFG in $E u S n_{3}$ compared to the other two compounds which is consistent with the experimental results obtained at nonzero temperatures. As EFG is very sensitive to the charge density around the nucleus, then its nearly constant value at Sn sites for all three compounds confirm the argument presented in Ref. [16] that the charge density around Sn nucleus are rather identical and independent of rare earth atoms. In Table IV we compare the 
TABLE III: The comparison of the derived EFG in $10^{21} \mathrm{~V} / \mathrm{m}^{2}$ from the measured quadrupole interactions of ${ }^{119} \mathrm{Sn}\left(\mathrm{Q}=0.124, E_{0}=23.8 \mathrm{keV}\right)$ at $\mathrm{T}=77 \mathrm{~K}$ and $4.2 \mathrm{~K}$ with the theoretical values calculated at $\mathrm{T}=\mathrm{OK}$ within $\mathrm{LDA}$, GGA without spin polarization (GGA), and GGA with spin polarization (GGA+SP).

\begin{tabular}{cccc}
\hline \hline & $\mathrm{SmSn}_{3}$ & $\mathrm{EuSn}_{3}$ & $\mathrm{GdSn}_{3}$ \\
\hline LDA & 17.49 & 16.56 & 16.51 \\
GGA & 16.71 & 15.35 & 15.39 \\
GGA+SP ${ }^{\mathrm{E}}{ }^{\text {Expt. }}{ }^{T=4.2 K}$ & 15.20 & 14.03 & 15.20 \\
${ }^{a}$ Expt. $^{T=77 K}$ & 13.82 & 13.56 & - \\
\hline \hline
\end{tabular}

${ }^{a}$ Ref. [16]

calculated EFG on Cd impurity with experiment [20]. Here also, the results obtained by GGA+SP are in better agreement with experiment. The EFG drop for Eu and Yb compounds, attributed to a changing valency, is clearly visible. The small deviation between calculation and experiment presented in Tables [II and IV are not unexpected given the uncertainties of the temperature effect, which are not included in our calculation. It seems that the electric field gradient at most cases decreases when the temperature rises. The EFG measured in $\mathrm{YbIn}_{3}$ and $\mathrm{EuSn}_{3}$ at two different temperatures given in Tables III and IV confirm this expectation. In addition to that, to avoid time consuming, we have not relaxed the atomic position. Since EFG may strongly be affected by local geometry, then the relaxation of the atoms from their original lattice positions can alter their value [21], hence the atomic unrelaxation can be considered as an another source of error in our calculation. The last possible source of error is that we have used experimental lattice parameter to calculate EFG which are not identical with the value obtained from E-V curves. Although we will show later that the effect of this discrepancy is ignorable.

At this stage we raise a question that our ab initio study is going to answer. To which extent the $\mathrm{EFG}$ at $\mathrm{X}$ in a $R X_{3}$ compound is sensitive to the behavior of $\mathrm{f}$ electrons in $\mathrm{R}$ and how the spin polarization can push the system in right direction? In order to tackle this question, we will follow Ref. [7] by introducing a visualization tool called anisotropy function $\Delta p(E)$. This function allows us a transparent interpretation of the qualitative behavior of Vzz. As Vzz expresses the deviation from spherical symmetry of the electron 

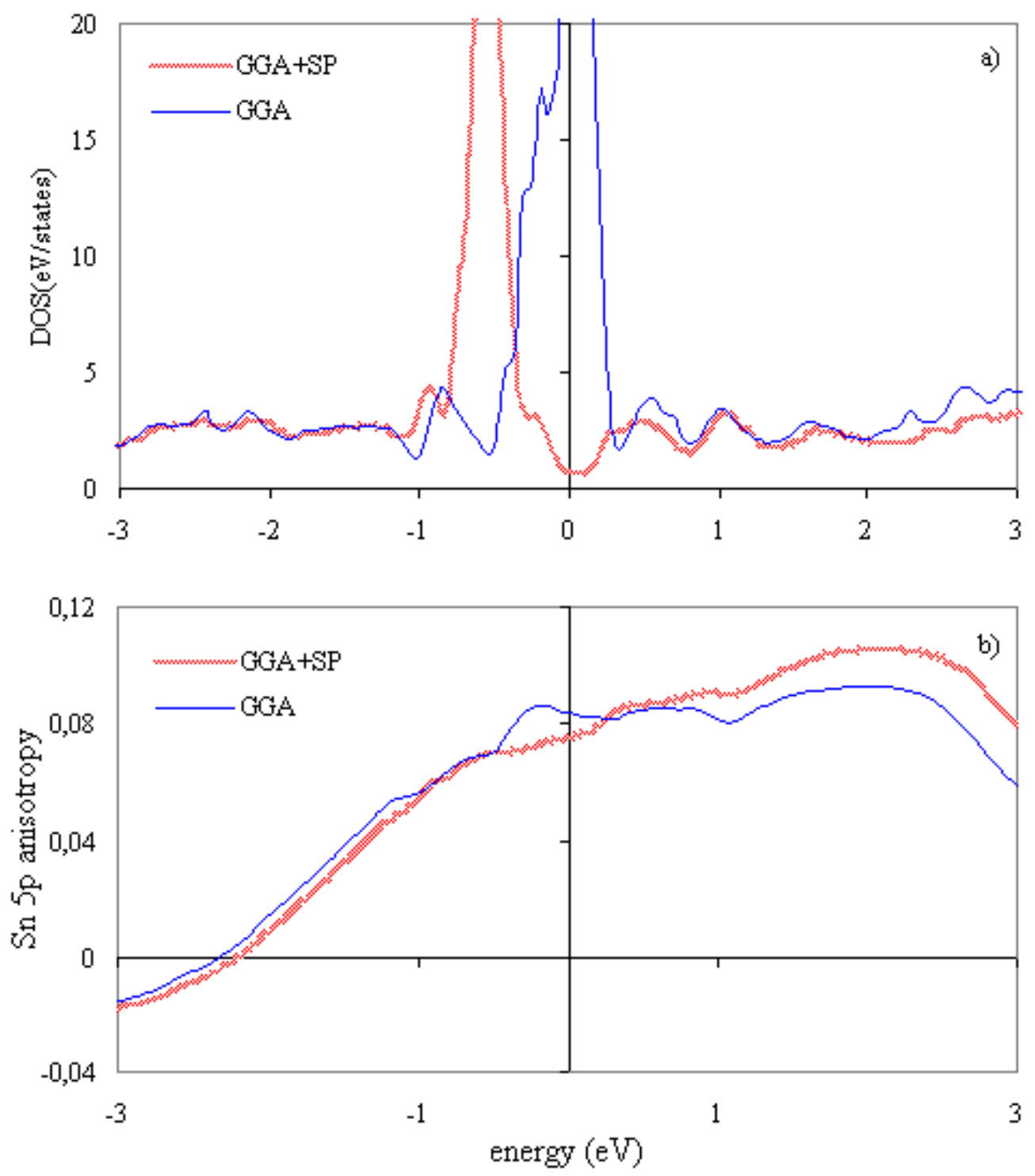

FIG. 1: All graphs are for EuSn3. The solid line is for GGA, and faint line for GGA+SP. (a) total DOS, (b) Sn 5p anisotropy function.

density in the environment of $\mathrm{X}$, hence this aspherically is given in terms of p-orbitals 

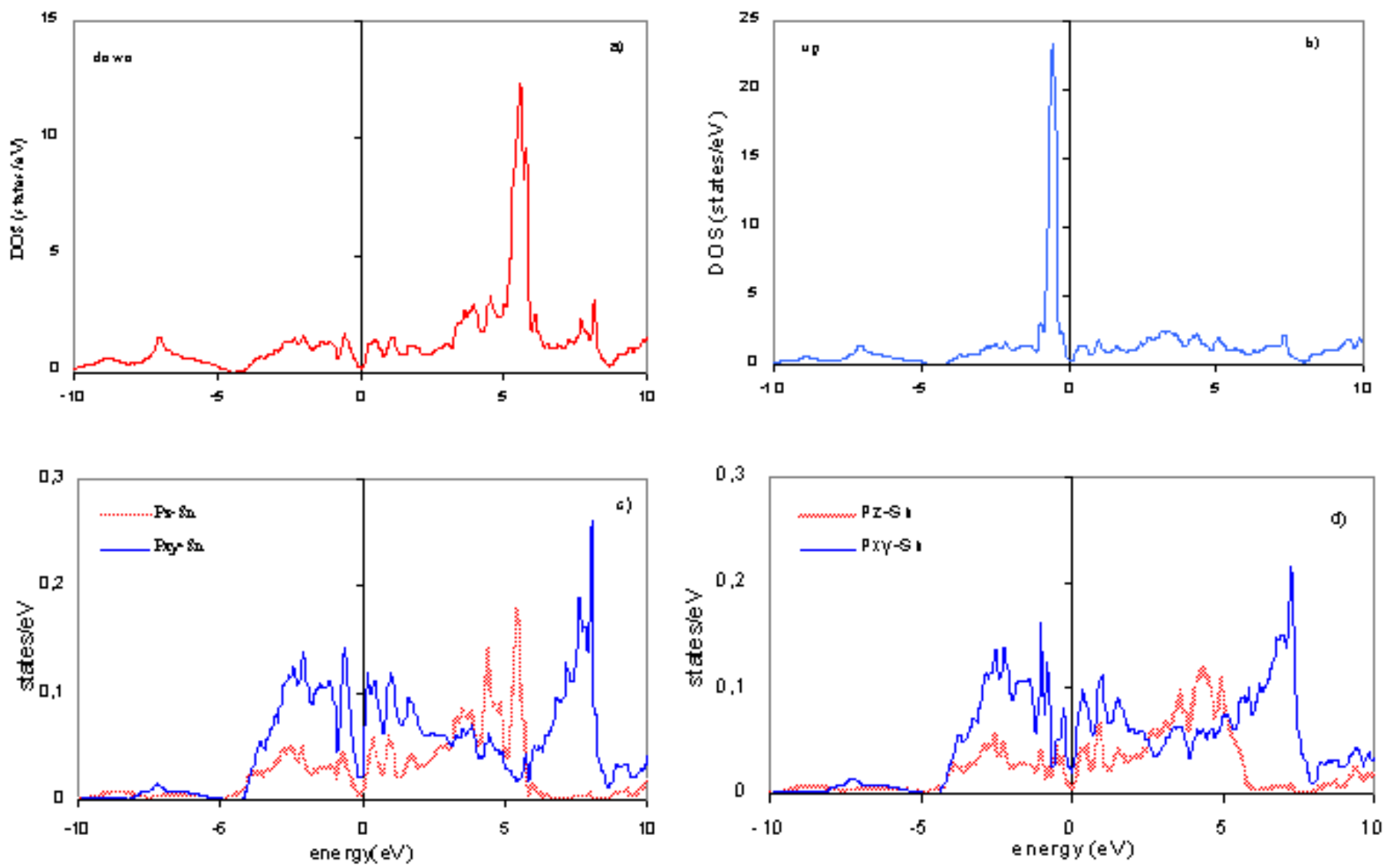

FIG. 2: All graphs are for EuSn3. (a) total spin-up DOS, (b) total spin-down DOS. The changes of $\mathrm{pz}$ (faint curves) and pxy (solid curves) as a function of energy are shown in (c) for spin-up and in (d) for spin-down.

TABLE IV: The comparison of the derived EFG in $10^{21} \mathrm{~V} / \mathrm{m}^{2}$ from the measured quadrupole coupling constants of ${ }^{111} \mathrm{Cd}\left(\mathrm{Q}=0.83 \mathrm{~b}, E_{0}=247 \mathrm{keV}\right)$ at $\mathrm{T}=300 \mathrm{~K}$ and $4.2 \mathrm{~K}$ with the theoretical values calculated at $\mathrm{T}=\mathrm{OK}$ within GGA without spin polarization (GGA), and GGA with spin polarization (GGA+SP).

\begin{tabular}{|c|c|c|c|c|c|c|}
\hline & $\mathrm{SmSn}_{3}$ & $\mathrm{EuSn}_{3}$ & $\mathrm{GdSn}_{3}$ & $\operatorname{TmIn}_{3}$ & $\mathrm{YbIn}_{3}$ & $\operatorname{LuIn}_{3}$ \\
\hline GGA & 4.06 & 3.44 & 3.64 & 3.36 & 3.11 & 6.08 \\
\hline $\mathrm{GGA}+\mathrm{SP}$ & 2.62 & 1.81 & 3.76 & 3.49 & 3.12 & 6.08 \\
\hline${ }^{a}$ Expt. ${ }^{T=300 K}$ & 2.12 & 0.63 & 2.12 & 4.37 & 1.92 & 4.32 \\
\hline${ }^{a} \operatorname{Expt.}^{T=4.2 K}$ & - & - & - & - & 2.16 & - \\
\hline
\end{tabular}

${ }^{a}$ Ref.[20] 
(s and d orbitals have ignorable effects [7]) by following expression:

$$
\begin{aligned}
V_{z z} \approx & V_{z z}^{p} \\
V_{z z}^{p}= & \Delta p\left(E_{F}\right)\left\langle\frac{1}{r^{3}}\right\rangle_{p} \\
& \Delta p\left(E_{F}\right)=\frac{1}{2} p_{x y}^{I}\left(E_{F}\right)-p_{z}^{I}\left(E_{F}\right) \\
& p_{i}^{I}\left(E_{1}\right)=\int_{-\infty}^{E_{1}} p_{i}(E) d E
\end{aligned}
$$

Here $\left\langle\frac{1}{r^{3}}\right\rangle_{p}$ is an expectation value for the p-orbitals, $p_{z}(E)$ is the partial p-DOS (Density of States) in the muffin tin sphere around an atom, and $E_{F}$ is the Fermi energy. The integral $p_{i}^{I}\left(E_{1}\right)$ counts the number of $p_{i}$ electrons in a muffin-tin sphere with energy less than $E_{1}$.

In order to investigate the effect of spin polarization on the value of EFG, the total DOS of EuSn3 within GGA and GGA+SP are shown in Fig1.a. The only considerable effect of spin polarization is the shift of f-peak from the initial fermi level to the left. Due to the hybridization of $\mathrm{f}$ elections on $\mathrm{R}$ sites with $\mathrm{p}$ electrons on neighboring $\mathrm{X}$ cites, the shift of $\mathrm{f}$ peak affect Pxy and $\mathrm{Pz}$ and subsequently $\Delta p\left(E_{F}\right)$. The changes of anisotropy function with energy are shown in Figl.b. It is clearly seen that $\Delta p\left(E_{F}\right)$ and as a result of that EFG obtained by GGA +SP is lower than the corresponding values within GGA.

To analyze the origin of the EFG on $\mathrm{Cd}$, Sn, and In sites, we decomposed the calculated EFG into several different contributions (Table $\mathrm{V}$ ). The valence EFG ( ${ }^{v a l} V_{z z}$ ) originates from the asphericity of the valence (and semicore) charge density inside the muffin tin sphere. The lattice EFG $\left({ }^{l a t} V_{z z}\right)$ arises from the boundary value problem and charge distribution outside the muffin-tin sphere. ${ }^{v a l} V_{z z}$ is further decomposed into spin up $\left({ }^{v a l} V_{z z}^{\uparrow}\right)$ and spin down $\left({ }^{v a l} V_{z z}^{\downarrow}\right)$ contributions. It is seen that the lattice contribution is negligibly small (less than $2 \%$ of the total EFG), which reflects the fact that the main origin of the EFG lies in the deviation from spherical symmetry of the valence charge density near the nucleus. It also confirm that the total EFG is not highly sensitive to the lattice parameters, hence the non-optimized experimental lattice constant used in our calculation is acceptable. ${ }^{v a l} V_{z z^{-}}^{\uparrow a l} V_{z z}^{\downarrow}$ is expected to be a qualitative criteria for magnetism of the system; the vanishing of this quality for non-magnetic compounds such as $\mathrm{YbIn}_{3}$ and $\mathrm{LuIn}_{3}$ confirm this expectation.

At the end to analyze the up and down contributions to Vzz on Cd, Sn and In in the listed compound we concentrate, as an example, on Sn site in $E u S n_{3}$. The spin up and 


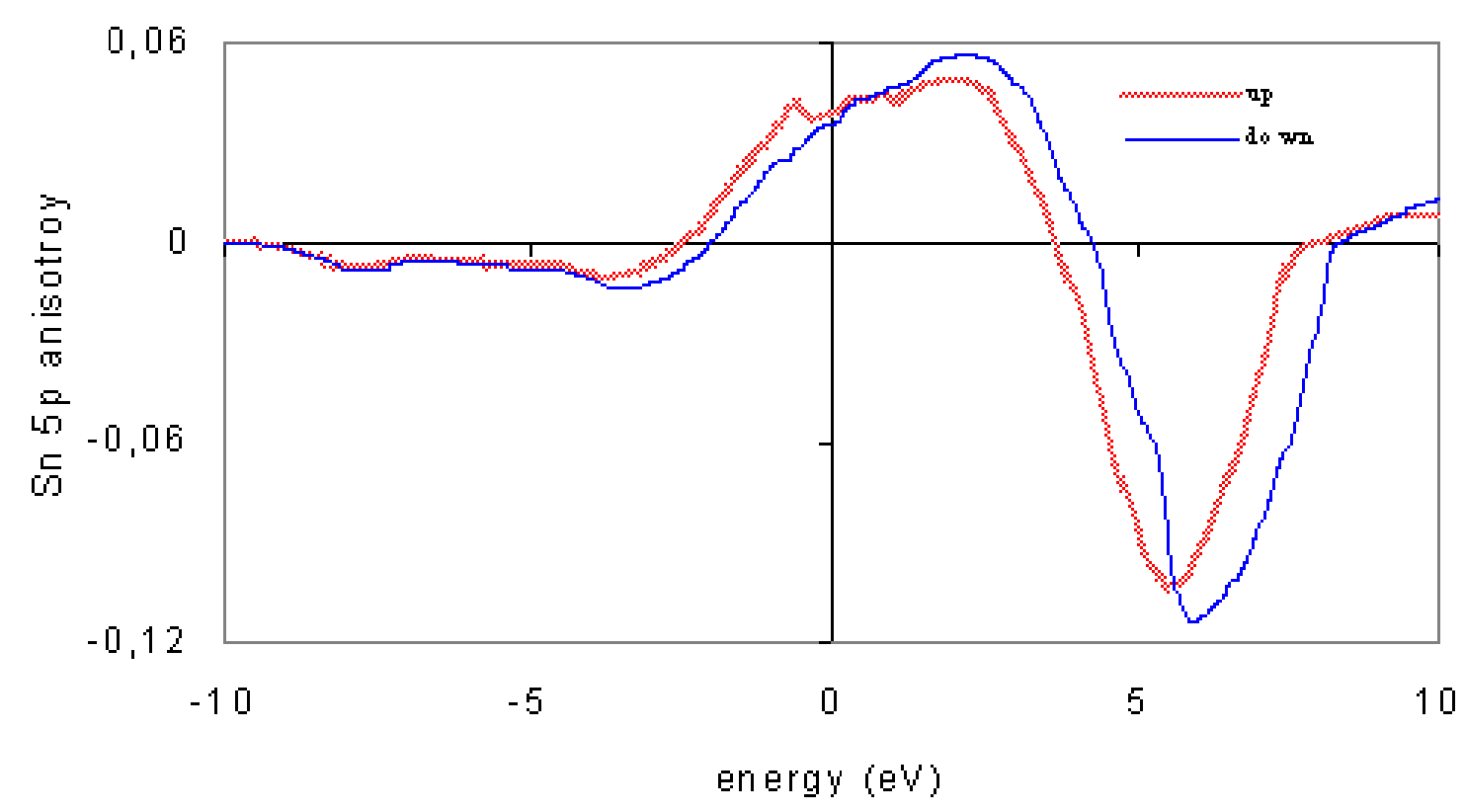

FIG. 3: The changes of anisotropy function $\Delta p(E)$ for both up (faint) and down (solid) spin directions with respect to energy.

down DOS and their corresponding $p_{z}$ and $p_{x y}$ on Sn cites are shown in Fig2. First of all, it is seen that spin polarization has splited the up and down $\mathrm{f}$ peaks (Fig2.a, and b). This feature is compatible with reality. In nature there are two types of $f$ electrons, localized and nonlocalized, and as a result of that the $\mathrm{f}$ band should split into two sub-bands. In regular GGA as the strong correlation between the f electrons are not treated properly, then all of them are located in a single peak at $E_{F}$. Hence it is not unjustified to expect that magnetism push the system in the right direction. How the f-peak splitting affects $p_{z}, p_{x y}, \Delta p\left(E_{F}\right)$, and consequently EFG? Fig2c and d show the changes of $p_{z}$ and $p_{x y}$ with energy for up and down spin separately. Below $-4 \mathrm{eV}$ the two set of curves coincide. In the region $[-4,0] \mathrm{eV} P_{x y}^{\uparrow}$ is slightly larger than $P_{x y}^{\downarrow}$. This difference is more visible at around $-1 \mathrm{eV}$, which is the location of $f^{\uparrow}$ peak. One can conclude that the $\mathrm{Sn} p^{\uparrow}$ electrons in the xy-plane hybridizes with the f-electrons of the neighboring rare earths (which are all 4 in the xy-plane). In Fig.3 the anisotropy function $\Delta p(E)$ is given for two spin directions (call it $\Delta p^{\uparrow}(E)$ and $\Delta p^{\downarrow}(E)$ ). At fermi level, $\Delta p^{\uparrow}(E)$ is slightly larger than $\Delta p^{\downarrow}(E)$. Exactly the same was seen for $V_{z z}$ in Table III, which demonstrates once more that the p-anisotropy really reflects the behavior of $V_{z z}$. As we already expected from $p_{z}$ and $P_{x y}$ curves, no difference is observed between the two curves below $-4 \mathrm{eV}$. In the 
TABLE V: The decompositions of calculated EFG's at Cd, Sn and In sites into the valence EFG for spin up and down and the total lattice EFG in $10^{21} \mathrm{~V} / \mathrm{m}^{2}$.

\begin{tabular}{lcccc}
\hline \hline & LuIn $_{3}$ & & & \\
\hline & $V_{z z}^{\text {latt }}$ & ${ }^{v a l} V_{z z}$ & ${ }^{v a l} V_{z z}^{\uparrow}$ & \\
& & $C d$ & & \\
$\mathrm{SmSn}_{3}$ & -0.03 & 2.62 & 2.26 \\
$\mathrm{EuSn}_{3}$ & -0.03 & 1.81 & 1.49 & 0.39 \\
$\mathrm{GdSn}_{3}$ & -0.04 & 3.76 & 2.18 & 0.35 \\
$\mathrm{TmIn}_{3}$ & -0.03 & 3.49 & 1.70 & 1.62 \\
$\mathrm{YbIn}_{3}$ & -0.00 & 3.12 & 1.56 & 1.82 \\
$\operatorname{LuSn}_{3}$ & -0.02 & 6.08 & 3.05 & 1.56 \\
& & $S n$ & & 3.05 \\
$\operatorname{SmSn}_{3}$ & -0.02 & 15.02 & 8.49 & 6.73 \\
$\operatorname{EuSn}_{3}$ & -0.03 & 14.03 & 7.33 & 6.73 \\
$\operatorname{GdSn}_{3}$ & -0.02 & 15.83 & 7.39 & 8.46 \\
& & In & & 4.74 \\
$\operatorname{TmIn}_{3}$ & -0.01 & 8.95 & 4.22 & 4.18 \\
$\mathrm{YbIn}_{3}$ & -0.01 & 8.34 & 4.18 & 5.62 \\
$\operatorname{LuIn}_{3}$ & -0.04 & 11.23 & 5.62 & \\
\hline \hline
\end{tabular}

region [-4, 0 ] $\Delta p^{\uparrow}(E)$ is larger than $\Delta p^{\downarrow}(E)$ and at around $-1 \mathrm{eV} p^{\uparrow}$ anisotropy shows a clear peak. This is due to the $f^{\uparrow}$ peak located in this area.

\section{CONCLUSION}

We employed the FP-LAPW method to calculate the structural properties of $\mathrm{RSn}_{3}$ ( $\mathrm{R}=\mathrm{Sm}, \mathrm{Eu}, \mathrm{Gd})$ and $\mathrm{RIn}_{3}(\mathrm{R}=\mathrm{Tm}, \mathrm{Yb}, \mathrm{Lu})$ by LDA, GGA, GGA+SP, and GGA+open core ( valence 2, 2.5, and 3 ) approaches. For $\mathrm{Eu}$ and $\mathrm{Yb}$ compounds open core and for other compounds GGA+SP results are in better agreement with experiment. We also calculated the EFG at $\mathrm{Sn}$, In, and impurity $\mathrm{Cd}$ sites in $\mathrm{RSn}_{3}(\mathrm{R}=\mathrm{Sm}, \mathrm{Eu}, \mathrm{Gd})$ and $\mathrm{RIn} \mathrm{n}_{3}(\mathrm{R}=\mathrm{Tm}$, $\mathrm{Yb}, \mathrm{Lu}$ ) compounds. Here also, the results obtained by GGA+SP are in better agreement with experiment. The reasonable agreement of the results calculated by GGA+SP with experiment confirm the fact that even for such highly correlated systems the spin splitting introduced by magnetism can compensate the strong coulomb correlation. Our 
approach has the advantage of treating the localized and itinerant electronic states on the same common footing.

\section{Acknowledgments}

S.J.A., and H.A. acknowledge the financial support of the Isfahan University of Technology and also the Association Scheme of the Abdus Salam International Centre for Theoretical Physics (ICTP). 
[1] P. Novak, F. Boucher, Gressier, P. Blaha, and K. Schwarz, Phys. Rev. B 63, 235114 (2001).

[2] D.L. Price, B.R. Cooper, Say-Peng Lim, I. Avgin, Phys. Rev. B 61, 9867 (2000).

[3] V.I. Anisimov, F. Aryasetiawan, and A.I. Lichtenstein, J. Phys.: Condens. Matter 9, 767 (1997).

[4] A. Svane, W.M. Temmerman, Z. Szotek, L. Petit, P. Strange, and H. Winter, Phy. Rev. B 62 , 13394 (2000).

[5] P. Strange, A. Svane, W.M. Temmerman, Z. Szotek, Nature, 399, 756 (1999).

[6] M. Richter, J. Phys. D: Appl. Phys. 31, 1017 (1998).

[7] S. Jalali Asadabadi, S. Cotteneer, H. Akbarzadeh, R. Saki, and M. Rots, submitted to PRB , (2002).

[8] P. Blaha, K. Schwarz, and P. H. Dederichs, Phys. Rev. B 37, 2792 (1988).

[9] M. Weinert, J. Math. Phys. 22, 2433 (1981).

[10] P. Blaha, K. Schwarz, and J. Luitz, WIEN97, A full Potential Linearized Augmented Plane Wave Package for Calculating Crystal Properties (Karlheinz Schwarz, Tech. Univ. Wien, Vienna 1999). ISBN 3-9501031-0-4. Updated version of P. Blaha, K. Schwarz, P. Sorantin, and S.B. Trickey, Comp. Phys. Commun. 59, 399 (1990). In this work, version 97.10 was used.

[11] D.J. Singh, Phys. Rev. B 43, 6388 (1991).

[12] E.L. Bominaar, J. Guillin, A. Sawayn, and A.X. Traufuxen, Phy. Rev. B 39, 72 (1989).

[13] H.J. Monkhurst, J.D. Pack, Phys. Rev. B 13, 5188 (1976).

[14] J.P. Perdew, A. Zuanger, Phys. Rev. B 23, 5048 (1981).

[15] J.P. Perdew, S. Burke, and M. Ernzerhof, Phys. Rev. Lett. 77, 3856 (1996).

[16] J.P. Sanchez, J.M. Friedt, G.K. Shenoy, A. Percheron, and J.C. Achard, J. Phys. C: Solid State Phys. 9, (1976).

[17] F. Birch, J. Geophys. Res. 83, 1257 (1978).

[18] A. Delin, L. Fast, and Börje Johanson, Phys. Rev. B 8, 58 (1998).

[19] L. Petit, A. Svane, W. M. Temmerman, and Z. Szotek, Phys. Rev. B 63, 165107 (2001).

[20] G.P. Schwartz, and D.A. Shirley, Hyperfine Interaction 3, 67 (1977).

[21] S. Lany, P. Blaha, J. Hamann, V. Ostheimer, H. Wolf, and T. Wichert, Phys. Rev. B 62, 2259 (2000). 